\title{
Prime power divisors of binomial coefficients
}

\author{
By J.W. Sander at Hannover
}

\section{Introduction}

Around 1850, Chebyshev was the first mathematician who proved any worthwhile results on the prime counting function $\pi(x)$, namely that it is bounded from above and below by $\frac{x}{\log x}$. This can be obtained by looking at the prime factors of the "middle" binomial coefficients $\left(\begin{array}{c}2 n \\ n\end{array}\right)$, because these coefficients have enormously many distinct prime factors. On the other hand, in 1975, Erdös [5] conjectured that for $n>4$, the binomial coefficient $\left(\begin{array}{c}2 n \\ n\end{array}\right)$ is never square-free. In a subsequent article, Erdös and Graham [3] asked more general questions (see also [4] and [6]), namely: Given a positive integer $a$, does for sufficiently large $n$, there exist a prime $p$ such that $p^{a} \mid\left(\begin{array}{c}2 n \\ n\end{array}\right)$ ? Does $p$ tend to infinity for increasing $n$ ? Do these properties also hold for binomial coefficients $\left(\begin{array}{c}2 n \pm d \\ n\end{array}\right)$, if $d$ is "not too large"?

The original conjecture of Erdös was settled by Sárközy [14] in 1985 for sufficiently large $n$. In [10], [11] and [13], the author gave answers to the second and third question mentioned above, using a new exponential sum estimate [12]. In this paper, we will finally give an answer to all three questions by proving the following

Theorem 1. Let $0<\varepsilon<1$, and $a \in \mathbb{N}$. Then there exists $m_{0}=m_{0}(\varepsilon, a)$ such that for all $m \geqq m_{0}$ and all $0 \leqq k \leqq m$ satisfying

$$
|m-2 k|<m^{1-\varepsilon},
$$

we have $p^{a} \mid\left(\begin{array}{l}m \\ k\end{array}\right)$ for some prime $p>\frac{1}{2} m^{\frac{1}{a+1}}$

The main tool for the proof of Theorem 1 will be an upper bound of the exponential sum 


$$
\sum_{p \leqq P} e\left(x\left(\frac{h_{1}}{p^{j_{1}}}+\ldots+\frac{h_{r}}{p^{j_{r}}}\right)\right),
$$

where $e(x)=e^{2 \pi i x}$ for real $x$, as usual. This generalizes the corresponding results obtained by Jutila [7] and the author [12]. As a corollary, we get an asymptotic formula for

$$
\operatorname{card}\left\{p \leqq P:\left\{\frac{x}{p^{j}}\right\}<\sigma_{j}(1 \leqq j \leqq J)\right\},
$$

where $0<\sigma_{j} \leqq 1$.

In a forthcoming paper, we will apply these exponential sum estimates to the method of Sárközy [14] in order to obtain upper and lower bounds for the highest $a$-th power dividing binomial coefficients.

\section{Preliminaries}

In the sequel, let $r$ be a positive integer and let real numbers $h_{i}(1 \leqq i \leqq r)$ and positive integers $j_{i}(1 \leqq i \leqq r)$ be given such that

$$
\begin{gathered}
h=h_{1} \geqq 1, \\
H=\max \left\{\left|h_{i}\right|: 1 \leqq i \leqq r\right\}
\end{gathered}
$$

and

$$
1 \leqq j=j_{1}<j_{2}<\ldots<j_{r} \leqq J
$$

where $J$ is a positive real number. We define

$$
\Lambda(X, Y)=\left(\frac{\log X}{\log Y}\right)^{2}
$$

All the explicit and implicit constants may only depend on $J$. We adopt the convention that the constant $c$, which always is assumed to be positive, may change its value within equations and inequalities. This enables us to write

$$
x^{1-c} \log x \ll x^{1-c},
$$

for instance.

We will make use of methods due to Vinogradov-Karacuba, van der Corput and Jutila [7].

Lemma 1 [8]. Let $N, P$ and $n \geqq 2$ be positive integers, let $f(x)$ be a real function having continuous $(n+1)$ th derivative in the interval $N \leqq x \leqq N+P$. Let $c_{0}, c_{1}, c_{2}, c_{3}$ and $c_{4}$ be positive constants satisfying $c_{0}<1, c_{1}<1, c_{2}+c_{4}<c_{1}$, let $t$ be an integer with $c_{0} n \leqq t \leqq n$, and integers $s_{i}(1 \leqq i \leqq t), 2 \leqq s_{i} \leqq n$, such that for $N \leqq x \leqq N+P$ the following inequalities hold: 
(4)

$$
\begin{gathered}
\left|\frac{1}{(n+1) !} f^{(n+1)}(x)\right| \leqq P^{-c_{1}(n+1)}, \\
P^{-c_{2} s_{i}} \leqq\left|\frac{1}{s_{i} !} f^{\left(s_{i}\right)}(x)\right| \leqq P^{-c_{3} s_{i}} \quad(1 \leqq i \leqq t) .
\end{gathered}
$$

Then for each positive integer $P_{1} \leqq P$ and

$$
S=\sum_{x=N}^{N+P_{1}-1} e(f(x))
$$

we have for some positive constants $A$ and $\gamma$

$$
|S| \leqq A P^{1-\gamma / n^{2}}
$$

Lemma 2. Let $T^{\prime}>2, T=T^{\prime} h$ such that

$$
0 \leqq P^{\prime}<P<T^{\frac{1}{j+1}}+\frac{1}{100 j^{3}}
$$

and

$$
P(\log P)^{J}>J H(3 \log T)^{J}
$$

Then

$$
\left|\sum_{x=P}^{P+P^{\prime}} e\left(T^{\prime}\left(\frac{h_{1}}{x^{j_{1}}}+\ldots+\frac{h_{r}}{x^{j_{r}}}\right)\right)\right| \ll P^{1-c \Lambda(P, T)} .
$$

Proof. Without loss of generality we may assume

$$
J>8
$$

and

$$
P>(2 J)^{150 J^{2}}
$$

We intend to apply Lemma 1 and put $N=P$,

$$
\begin{gathered}
f(x)=T^{\prime}\left(\frac{h_{1}}{x^{j_{1}}}+\ldots+\frac{h_{r}}{x^{j_{r}}}\right), \\
n=\left[100 j^{2} \frac{\log T}{\log P}\right], \\
c_{0}=\frac{1}{500 j^{5}}, \quad c_{1}=1-\frac{1}{50 j^{2}}, \quad c_{2}=1-\frac{1}{25 j^{2}}, \quad c_{3}=\frac{1}{20 j^{2}}, \quad c_{4}=\frac{1}{60 j^{2}}
\end{gathered}
$$

and

$$
\left\{s_{i}: 1 \leqq i \leqq t\right\}=\left\{s \in \mathbb{N}:\left(1+\frac{1}{5 j^{2}}\right) \frac{\log T}{\log P} \leqq s \leqq\left(1+\frac{1}{j}\right) \frac{\log T}{\log P}\right\}
$$


By (6), we have

$$
\frac{\log T}{\log P}>\left(\frac{1}{j+1}+\frac{1}{100 j^{3}}\right)^{-1}
$$

Therefore

$$
\left(\left(1+\frac{1}{j}\right)-\left(1+\frac{1}{5 j^{2}}\right)\right) \frac{\log T}{\log P}>1+\frac{1}{3 j}>1,
$$

thus $\left\{s_{i}\right\} \neq \emptyset$. Moreover, (12) yields

$$
t \geqq\left(\left(1+\frac{1}{j}\right)-\left(1+\frac{1}{5 j^{2}}\right)\right) \frac{\log T}{\log P}-1>c_{0} 100 j^{2} \frac{\log T}{\log P} \geqq c_{0} n
$$

Obviously, $2 \leqq s_{i} \leqq n$. It remains to check (4) and (5) in Lemma 1. For $x>0$ and $m \in \mathbb{N}$, we have

$$
\frac{1}{m !} f^{(m)}(x)=(-1)^{m} \frac{T^{\prime}}{m !} \sum_{i=1}^{r} \frac{j_{i}\left(j_{i}+1\right) \cdots\left(j_{i}+m-1\right) h_{i}}{x^{j_{i}+m}}
$$

(7) and (6) give $P>H$. By (2) and (7), we thus get

$$
\frac{h}{P^{j}} \geqq \frac{\left|h_{i}\right|}{P^{j_{i}}} \quad(1 \leqq i \leqq r),
$$

which implies for $P=N \leqq x \leqq N+P=2 P$ with (9)

$$
\begin{aligned}
\left|\frac{1}{(n+1) !} f^{(n+1)}(x)\right| & \leqq J^{n+1} T^{\prime}\left(\frac{\left|h_{1}\right|}{P^{j_{1}+n+1}}+\cdots+\frac{\left|h_{r}\right|}{P^{j_{r}+n+1}}\right) \leqq \frac{J^{n+1} T^{\prime}}{P^{n+1}} r \frac{h}{P^{j}} \\
& \leqq \frac{J^{n+2} T}{P^{j+n+1}}=P^{(n+2) \frac{\log J}{\log P}+\frac{\log T}{\log P}-(j+n+1)}=P^{-c_{1}^{\prime}(n+1)}
\end{aligned}
$$

where

$$
c_{1}^{\prime}=1+\frac{j}{n+1}-\frac{(n+2) \log J}{(n+1) \log P}-\frac{\log T}{(n+1) \log P}>c_{1}
$$

by (9) and (10). Hence (4) holds.

In the same way, we get for $P \leqq x \leqq 2 P$ and $s \in\left\{s_{i}\right\}$ by (11)

$$
\left|\frac{1}{s !} f^{(s)}(x)\right| \leqq \frac{J^{s+1} T}{P^{j+s}}=P^{-c_{3}^{\prime} s},
$$

where

$$
c_{3}^{\prime}=1+\frac{j}{s}-\frac{(s+1) \log J}{s \log P}-\frac{\log T}{s \log P}>c_{3} .
$$


This proves the upper bound in (5). By (13), we have for $P \leqq x \leqq 2 P$ and $s \in\left\{s_{i}\right\}$ using (2)

$$
\left|\frac{1}{s !} f^{(s)}(x)\right| \geqq T^{\prime}\left(\frac{h}{x^{j+s}}-(r-1)\left(\begin{array}{c}
J-1+s \\
s
\end{array}\right) \frac{H}{x^{j_{2}+s}}\right) .
$$

We apply a weak form of Stirling's formula, namely

$$
\left(n+\frac{1}{2}\right) \log n-n+\frac{3}{2}\left(1-\log \frac{3}{2}\right) \leqq \log n ! \leqq\left(n+\frac{1}{2}\right) \log n-n+1
$$

For $J-1<s$, this implies together with (8)

$$
\left(\begin{array}{c}
J-1+s \\
s
\end{array}\right)<s^{J-1}
$$

For $J-1 \geqq s$, we have trivially

$$
\left(\begin{array}{c}
J-1+s \\
s
\end{array}\right)<4^{J-1}
$$

By (15), (16), (17), (2) and (3), we get for $s_{0}=\max (s, 4)$

$$
\begin{aligned}
\left|\frac{1}{s !} f^{(s)}(x)\right| & \geqq \frac{T^{\prime}}{x^{j+s}}\left(h-\frac{J s_{0}^{J-1} H}{x^{j_{2}-j}}\right) \\
& \geqq \frac{T^{\prime}}{(2 P)^{j+s}}\left(h-\frac{J s_{0}^{J-1} H}{P^{j_{2}-j}}\right) \\
& \geqq \frac{T^{\prime}}{(2 P)^{j+s}}\left(h-\frac{J s_{0}^{J-1} H}{P}\right) .
\end{aligned}
$$

By (7), (11) and (12)

$$
P>2 J s_{0}^{J} H
$$

Therefore, (18) yields

$$
\left|\frac{1}{s !} f^{(s)}(x)\right| \geqq \frac{T^{\prime} h}{2(2 P)^{j+s}}=P^{-c_{2}^{\prime} s}
$$

where

$$
c_{2}^{\prime}=\left(1+\frac{j}{s}\right)\left(1+\frac{\log 2}{\log P}\right)+\frac{\log 2}{s \log P}-\frac{\log T}{s \log P} \leqq c_{2}
$$

using (11), (9) and (12). Hence the lower bound in (5) also holds. This proves Lemma 2. 
Lemma 3. Let $T^{\prime}>2, T=T^{\prime} h$ and $0 \leqq P^{\prime}<P$ such that

$$
P \geqq T^{\frac{1}{j+1}}+\frac{1}{100 j^{3}}
$$

and

$$
P>2 J^{3} H
$$

\section{Then}

$$
\left|\sum_{x=P}^{P+P^{\prime}} e\left(T^{\prime}\left(\frac{h_{1}}{x^{j_{1}}}+\cdots+\frac{h_{r}}{x^{j_{r}}}\right)\right)\right| \ll \frac{P^{j+1}}{T} .
$$

Proof. We use van der Corput's well-known method. Again let

$$
f(x)=T^{\prime}\left(\frac{h_{1}}{x^{j_{1}}}+\cdots+\frac{h_{r}}{x^{j_{r}}}\right)
$$

We assume without loss of generality

$$
P>(2 J)^{200 J^{2}} \text {. }
$$

For $x>0$,

$$
f^{\prime}(x)=-T^{\prime}\left(\frac{j_{1} h_{1}}{x^{j_{1}+1}}+\cdots+\frac{j_{r} h_{r}}{x^{j_{r}+1}}\right) .
$$

Since by (2), (3) and (20) for $P \leqq x \leqq 2 P$

$$
\begin{aligned}
f^{\prime \prime}(x) & =T^{\prime}\left(\frac{j_{1}\left(j_{1}+1\right) h_{1}}{x^{j_{1}+2}}+\cdots+\frac{j_{r}\left(j_{r}+1\right) h_{r}}{x^{j_{r}+2}}\right) \\
& \geqq \frac{T^{\prime}}{x^{j_{2}+2}}\left(j(j+1) h x^{j_{2}-j}-(r-1) J(J+1) H\right) \\
& \geqq \frac{T^{\prime}}{(2 P)^{j_{2}+2}}\left(2 h P-J^{3} H\right)>0,
\end{aligned}
$$

$f^{\prime}(x)$ obviously is an increasing function. Because of (14), we get for $P \leqq x \leqq P+P^{\prime}$ by (19) and (21)

$$
\begin{aligned}
\left|f^{\prime}(x)\right| & \leqq \frac{T^{\prime} J}{P}\left(\frac{h_{1}}{P^{j_{1}}}+\cdots+\frac{h_{r}}{P^{j_{r}}}\right) \leqq \frac{T^{\prime} J}{P} r \frac{h}{P^{j}} \leqq \frac{J^{2} T}{P^{j+1}} \\
& <J^{2} P^{-\frac{1}{100 j}}<J^{2}(2 J)^{-\frac{2 J}{j}} \leqq \frac{1}{4}<1 .
\end{aligned}
$$


Thus Lemma 4.8 in [15] implies

$$
\sum_{x=P}^{P+P^{\prime}} e\left(T^{\prime}\left(\frac{h_{1}}{x^{j_{1}}}+\cdots+\frac{h_{r}}{x^{j_{r}}}\right)\right)=\int_{P}^{P+P^{\prime}} e\left(T^{\prime}\left(\frac{h_{1}}{x^{j_{1}}}+\cdots+\frac{h_{r}}{x^{j_{r}}}\right)\right) d x+O(1)
$$

The function $f^{\prime}(x)$ is increasing in $\left[P, P+P^{\prime}\right]$ and, by (3) and (20), we have in this interval

$$
\begin{aligned}
-f^{\prime}(x) & \geqq T^{\prime}\left(\frac{j h}{x^{j+1}}-(r-1) \frac{J H}{x^{j_{2}+1}}\right) \\
& \geqq \frac{T^{\prime}}{(2 P)^{j+1}}\left(j h-\frac{J^{2} H}{P^{j_{2}-j}}\right) \\
& \geqq \frac{1}{2} \frac{T^{\prime} h}{(2 P)^{j+1}} \geqq \frac{1}{2} \frac{T}{(2 P)^{j+1}}
\end{aligned}
$$

Hence Lemma 4.2 in [15] gives

$$
\left|\int_{P}^{P+P^{\prime}} e\left(T^{\prime}\left(\frac{h_{1}}{x^{j_{1}}}+\cdots+\frac{h_{r}}{x^{j_{r}}}\right)\right) d x\right| \ll \frac{P^{j+1}}{T}
$$

By (22), the desired result follows.

Lemma 4. Let $T^{\prime}>2,0 \leqq P^{\prime}<P$ and

$$
P>J H\left(3 \log T^{\prime} H\right)^{J} .
$$

Then

$$
\left|\sum_{x=P}^{P+P^{\prime}} e\left(T^{\prime}\left(\frac{h_{1}}{x^{j_{1}}}+\cdots+\frac{h_{r}}{x^{j_{r}}}\right)\right)\right| \ll P^{1-c \Lambda\left(P, T^{\prime} H\right)}+\frac{P^{j+1}}{T^{\prime}}
$$

Proof. For $P \geqq T^{\prime}$, the lemma obviously holds. In case $P<T^{\prime},(23)$ implies (7) and (20). Hence the proof is completed by Lemma 2 and Lemma 3.

Lemma 5. Let $x>2$. Then

$$
\left|\sum_{m \leqq M} e\left(x\left(\frac{h_{1}}{m^{j_{1}}} \cdots+\frac{h_{r}}{m^{j_{r}}}\right)\right)\right| \ll M^{1-c \Lambda(M, x H)}+M^{j+1} x^{-1}+H^{2}(\log x H)^{2 J} .
$$

Proof. For $M \leqq J^{2} H^{2}(3 \log x H)^{2 J}$, the lemma is obvious. Thus we assume

$$
M>J^{2} H^{2}(3 \log x H)^{2 J} .
$$


Let $\frac{1}{2} \leqq \kappa<1$. Then

$$
\begin{aligned}
\left|\sum_{m \leqq M} e\left(x\left(\frac{h_{1}}{m^{j_{j}}}+\cdots+\frac{h_{r}}{m^{j_{r}}}\right)\right)\right| & \leqq \sum_{m \leqq M^{\kappa}}\left|+\sum_{\substack{v \geqq 0 \\
M^{\kappa} 2^{v} \leqq M}}\right| \sum_{M^{\kappa} 2^{v} \leqq m<\min \left(M^{\kappa} 2^{v+1}, M\right)} \mid \\
& \leqq M^{\kappa}+\sum_{v} R_{v},
\end{aligned}
$$

where

$$
R_{\mathrm{v}}=\left|\sum_{M^{\kappa} 2^{v} \leqq m<\min \left(M^{\kappa} 2^{v+1}, M\right)} e\left(x\left(\frac{h_{1}}{m^{j_{1}}}+\cdots+\frac{h_{r}}{m^{j_{r}}}\right)\right)\right| .
$$

By Lemma 4 , (24), and since $\kappa \geqq \frac{1}{2}$, we get

$$
\begin{aligned}
R_{v} & \ll\left(M^{\kappa} 2^{v}\right)^{1-c \Lambda\left(M^{\kappa} 2^{v}, x H\right)}+\left(M^{\kappa} 2^{v}\right)^{j+1} x^{-1} \\
& \ll 2^{v} M^{\kappa-c \kappa^{3} \Lambda(M, x H)}+2^{(j+1) v} M^{(j+1) \kappa} x^{-1} .
\end{aligned}
$$

In $\sum R_{v}$, the variable $v$ runs through the interval $0 \leqq v \leqq \frac{(1-\kappa) \log M}{\log 2}$, therefore

$$
\sum 2^{v} \leqq 2 M^{1-\kappa}
$$

and

$$
\sum 2^{(j+1) v} \leqq 2^{j+1} M^{(j+1)(1-\kappa)}
$$

For large $\kappa<1$, the lemma follows.

We would like to mention that Lemma 5 is non-trivial only for $x \geqq M^{j}$.

Lemma 6. Let $2 \leqq M \leqq M^{\prime} \leqq \min (2 M, N) \leqq N \leqq x$ and $B \geqq 2$. Then

$$
\begin{aligned}
T & :=\sum_{M<m \leqq M^{\prime}}\left|\sum_{B<n \leqq \frac{N}{m}} \Lambda(n) e\left(x\left(\frac{h_{1}}{(m n)^{j_{1}}}+\cdots+\frac{h_{r}}{(m n)^{j_{r}}}\right)\right)\right|^{2} \\
& \ll\left(N^{2} M^{-1-c \Lambda(M, x H)}+N^{j+2}(M x)^{-1}+N+N^{2} M^{-2} H\right)(\log x H)^{J+2},
\end{aligned}
$$

where $\Lambda(n)$ denotes von Mangoldt's function.

Proof. For $M \leqq J H(3 \log x H)^{J}$, we obviously have

$$
\begin{aligned}
T & \leqq J H(3 \log x H)^{J}\left(\frac{N}{M} \log N\right)^{2} \\
& \ll N^{2} M^{-2} H(\log x H)^{J+2},
\end{aligned}
$$

which proves the lemma in this case. Hence, let 


$$
M>J H(3 \log x H)^{J} .
$$

Clearly

$$
\begin{aligned}
T & =\sum_{M<m \leqq M^{\prime}} \sum_{B<n_{1} \leqq \frac{N}{m}} \sum_{B<n_{2} \leqq \frac{N}{m}} \Lambda\left(n_{1}\right) \Lambda\left(n_{2}\right) e\left(x \sum_{i=1}^{r}\left(\frac{h_{i}}{\left(m n_{1}\right)^{j_{i}}}-\frac{h_{i}}{\left(m n_{2}\right)^{j_{i}}}\right)\right) \\
& =\sum_{B<n_{1} \leqq \frac{N}{M}} \sum_{B<n_{2} \leqq \frac{N}{M}} \Lambda\left(n_{1}\right) \Lambda\left(n_{2}\right) \sum_{\substack{M<m \leqq M^{\prime} \\
m \leqq \frac{N}{n_{1}}, m \leqq \frac{N}{n_{2}}}} e\left(x\left(\frac{h_{1} \Delta_{1}}{m^{j_{1}}}+\cdots+\frac{h_{r} \Delta_{r}}{m^{j_{r}}}\right)\right)
\end{aligned}
$$

with

$$
\Delta_{i}=\left(\frac{1}{n_{1}^{j_{i}}}-\frac{1}{n_{2}^{j_{i}}}\right) \quad(1 \leqq i \leqq r)
$$

Thus

$$
\begin{aligned}
T & \ll(\log N)^{2} \sum_{n_{1} \leqq \frac{N}{M}} \sum_{n_{2} \leqq \frac{N}{M}}\left|\sum_{\substack{M<m \leqq M^{\prime} \\
m \leqq \frac{N}{n_{1}}, m \leqq \frac{N}{n_{2}}}} e\left(x\left(\frac{h_{1} \Delta_{1}}{m^{j_{1}}}+\cdots+\frac{h_{r} \Delta_{r}}{m^{j_{r}}}\right)\right)\right| \\
& \left.\ll(\log N)^{2}\left(N+\sum_{0<n_{1}<n_{2} \leqq \frac{N}{M}} \mid \sum_{M<n \leqq M^{\prime}} e\left(x\left(\frac{h_{1} \Delta_{1}}{m^{j_{1}}}+\cdots+\frac{h_{r} \Delta_{r}}{m^{j_{r}}}\right)\right)\right)\right) \\
& =(\log N)^{2}\left(N+T_{1}\right),
\end{aligned}
$$

say. By the mean value theorem, we have for fixed $1<n_{1}<n_{2} \leqq \frac{N}{M}$

$$
0<\Delta_{r}<\cdots<\Delta_{1} \leqq 1
$$

By this and (25), Lemma 4 implies

$$
\text { (27) } \begin{aligned}
T_{1} & =\sum_{1<n_{1}<n_{2} \leqq \frac{N}{M}}\left|\sum_{M<m \leqq M^{\prime}} e\left(x \Delta_{1}\left(\frac{h_{1}}{m^{j_{1}}}+\frac{h_{2} \Delta_{2} / \Delta_{1}}{m^{j_{2}}}+\cdots+\frac{h_{r} \Delta_{r} / \Delta_{1}}{m^{j_{r}}}\right)\right)\right| \\
& \ll \sum_{1<n_{1}<n_{2} \leqq \frac{N}{M}}\left(M^{1-c \Lambda(M, x H)}+M^{j+1}\left(x \Delta_{1}\right)^{-1}\right) \\
& \ll N^{2} M^{-1-c \Lambda(M, x H)}+M^{j+1} x^{-1} \underset{1<n_{1}<n_{2} \leqq \frac{N}{M}}{\sum} \frac{1}{\Delta_{1}} .
\end{aligned}
$$

We set $\Delta_{0}=n_{2}-n_{1}$. For $1<n_{1}<n_{2}$ and $1 \leqq i \leqq r$, we get

$$
\Delta_{i}=\frac{n_{2}^{j_{i}}-n_{1}^{j_{i}}}{\left(n_{1} n_{2}\right)^{j_{i}}}=\Delta_{0} \frac{n_{2}^{j_{i}-1}+n_{2}^{j_{i}-2} n_{1}+\cdots+n_{2} n_{1}^{j_{i}-2}+n_{1}^{j_{i}-1}}{\left(n_{1} n_{2}\right)^{j_{i}}} \geqq \frac{\Delta_{0} j_{i}}{n_{2}^{j_{i}+1}}
$$


Therefore,

$$
\begin{aligned}
\sum_{1<n_{1}<n_{2} \leqq \frac{N}{M}} \frac{1}{\Delta_{1}} & =\sum_{\substack { 0<\Delta_{0}<\frac{N}{M} \\
\begin{subarray}{c}{1<n_{1}<n_{2} \leqq \frac{N}{M} \\
n_{2}-n_{1}=\Delta_{0}{ 0 < \Delta _ { 0 } < \frac { N } { M } \\
\begin{subarray} { c } { 1 < n _ { 1 } < n _ { 2 } \leqq \frac { N } { M } \\
n _ { 2 } - n _ { 1 } = \Delta _ { 0 } } }\end{subarray}} \frac{1}{\Delta_{1}} \\
& \leqq \frac{1}{j_{1}} \sum_{0<\Delta_{0}<\frac{N}{M}} \frac{1}{\Delta_{0}} \sum_{\substack{0<n_{2} \leqq \frac{N}{M} \\
n_{2}^{j+1}}} \\
& \ll\left(\frac{N}{M}\right)^{j+2} \log N .
\end{aligned}
$$

By (27),

$$
T_{1} \ll N^{2} M^{-1-c \Lambda(M, x H)}+N^{j+2}(M x)^{-1} \log N
$$

This and (26) yield the desired result.

\section{Vaughan's identity}

The application of Vaughan's identity instead of Vinogradov's rather complicated combinatorial argument is by now a well-known technique in analytic number theory. In our case, it simplifies the method of Jutila [7]. we have

As a corollary to Vaughan's identity (see for instance [16], [17], or [2], p. 138-140),

Lemma 7. Let $U \geqq 2, V \geqq 2, U V \leqq N$, and let $f(x)$ be a complex-valued function satisfying $|f(x)|=1$ for real $x$. Then

$$
\sum_{n \leqq N} \Lambda(n) f(n) \ll V+(\log N) S_{1}+S_{2},
$$

where $\Lambda(n)$ denotes von Mangoldt's function, and

$$
\begin{aligned}
& S_{1}=\sum_{t \leqq U V} \max _{w>0}\left|\sum_{w \leqq s \leqq \frac{N}{t}} f(s t)\right|, \\
& S_{2}=\sum_{U<m<\frac{N}{V}} \sum_{V<n \leqq \frac{N}{m}} \sum_{\substack{d \leqq U \\
d \mid m}} \mu(d) \Lambda(n) f(m n) .
\end{aligned}
$$

Lemma 8. Let $x>0$ and $2 \leqq N \leqq x^{1 / j}$. Then

$$
\sum_{n \leqq N} \Lambda(n) e\left(x\left(\frac{h_{1}}{n^{j_{1}}}+\cdots+\frac{h_{r}}{n^{j_{r}}}\right)\right) \ll\left(N^{1-c \Lambda(N, x H)}+N^{\frac{j+2}{2}} x^{-\frac{1}{2}}+N^{\frac{5}{6}} H^{2}\right)(\log x H)^{4 J} .
$$


Proof. We apply Lemma 7 with

$$
f(n)=e\left(x\left(\frac{h_{1}}{n^{j_{1}}}+\cdots+\frac{h_{r}}{n^{j_{r}}}\right)\right)
$$

First consider $S_{2}$. By splitting up the sum $\sum_{m}$ into intervals $M \leqq m<2 M$, we get

$$
S_{2} \ll(\log N) \max _{U<M<M^{\prime} \leqq \min \left(2 M, \frac{N}{V}\right)}\left|\sum_{M \leqq m<M^{\prime}}\left(\sum_{V<n \leqq \frac{N}{m}} \Lambda(n) f(m n)\right)\left(\sum_{\substack{d \leq U \\ d \backslash m}} \mu(d)\right)\right| .
$$

Cauchy's inequality implies

$$
\begin{aligned}
\left|\sum_{M \leqq m<M^{\prime}}\right| & \leqq\left(\sum_{m}\left|\sum_{V<n \leqq \frac{N}{m}} \Lambda(n) f(m n)\right|^{2}\right)^{1 / 2}\left(\sum_{m}\left(\sum_{\substack{d \leqq U \\
d \mid m}} \mu(d)\right)^{2}\right)^{1 / 2} \\
& =T_{1}^{1 / 2} T_{2}^{1 / 2},
\end{aligned}
$$

say. By Lemma 6,

$$
T_{1} \ll\left(N^{2} M^{-1-c \Lambda(M, x H)}+N^{j+2}(M x)^{-1}+N+N^{2} M^{-2} H\right)(\log x H)^{J+2} .
$$

Moreover,

$$
\begin{aligned}
T_{2} & \leqq \sum_{M \leqq m<M^{\prime}}\left(\sum_{\substack{d \leqq U \\
d\lceil m}} 1\right)^{2}=\sum_{d_{1} \leqq U} \sum_{d_{2} \leqq U} \sum_{\substack{M \leqq m<M^{\prime} \\
m \equiv 0 \bmod d_{1}, m=0 \bmod d_{2}}} 1 \\
& \leqq 2 M \sum_{d_{1} \leqq U} \sum_{d_{2} \leqq U} \frac{\left(d_{1}, d_{2}\right)}{d_{1} d_{2}} \leqq 2 M \sum_{b \leqq U} b \sum_{\substack{d_{1} \leqq U \\
d_{1} \leqq 0 \bmod b \quad}} \sum_{\substack{d_{2} \leqq U \\
d_{2} \cong 0 \bmod b}} \frac{1}{d_{1} d_{2}} \\
& \ll M(\log N)^{3} .
\end{aligned}
$$

Together, we get

$$
\begin{aligned}
S_{2} & \ll(\log N) \max _{U<M \leqq \frac{N}{V}}\left(T_{1} T_{2}\right)^{1 / 2} \\
& \ll(\log x H)^{\frac{J+7}{2}} \max _{U<M \leqq \frac{N}{V}}\left(N M^{-c \Lambda(M, x H)}+N^{\frac{j+2}{2}} x^{-\frac{1}{2}}+(N M)^{\frac{1}{2}}+N M^{-\frac{1}{2}} H^{\frac{1}{2}}\right) \\
& \ll\left(N U^{-c \Lambda(U, x H)}+N^{\frac{j+2}{2}} x^{-\frac{1}{2}}+N V^{-\frac{1}{2}}+N U^{-\frac{1}{2}} H^{\frac{1}{2}}\right)(\log x H)^{4 J}
\end{aligned}
$$


It remains to bound $S_{1}$. For $1 \leqq w \leqq \frac{N}{t}$, we have by Lemma 5

$$
\begin{aligned}
\sum_{w \leqq s \leqq \frac{N}{t}} e\left(x\left(\frac{h_{1}}{(s t)^{j_{1}}}+\cdots+\frac{h_{r}}{(s t)^{j_{r}}}\right)\right)= & \sum_{w \leqq s \leqq \frac{N}{t}} e\left(\frac{x}{t^{j_{1}}}\left(\frac{h_{1}}{s^{j_{1}}}+\frac{h_{2} t^{j_{1}-j_{2}}}{s^{j_{2}}}+\cdots+\frac{h_{r} t^{j_{1}-j_{r}}}{s^{j_{r}}}\right)\right) \\
(29) \quad \ll & \left(\frac{N}{t}\right)^{1-c \Lambda\left(\frac{N}{t}, x H\right)}+\left(\frac{N}{t}\right)^{j+1}\left(\frac{x}{t^{j}}\right)^{-1} \\
& +w^{1-c \Lambda(w, x H)}+H^{2}(\log x H)^{2 J} .
\end{aligned}
$$

The function

$$
g(y)=y^{1-c^{\prime}(\log y)^{2}}
$$

is increasing for $1 \leqq y<\exp \left(\left(3 c^{\prime}\right)^{-1 / 2}\right)$. Without loss of generality we may assume that for $c$ in (29), we have $c \leqq \frac{j^{2}}{3}$. Then for $N \leqq x^{1 / j}$

$$
\frac{N}{t} \leqq(x H)^{1 / j}=\exp \left(\frac{\log x H}{j}\right) \leqq \exp \left(\left(\frac{3 c}{(\log x H)^{2}}\right)^{-1 / 2}\right)
$$

Hence, for $N \leqq x^{1 / j}$, we get by (29)

$$
\begin{aligned}
\sum_{w \leqq s \leqq \frac{N}{t}} e\left(x\left(\frac{h_{1}}{(s t)^{j_{1}}}+\cdots+\frac{h_{r}}{(s t)^{j_{r}}}\right)\right) & \ll\left(\frac{N}{t}\right)^{1-c \Lambda\left(\frac{N}{t}, x H\right)}+\left(\frac{N}{t}\right)^{j+1} x^{-1} t^{j}+H^{2}(\log x H)^{2 J} \\
& =\left(\frac{N}{t}\right)^{1-c \Lambda\left(\frac{N}{t}, x H\right)}+N^{j+1}(x t)^{-1}+H^{2}(\log x H)^{2 J}
\end{aligned}
$$

thus

$$
\begin{aligned}
\max _{w>0}\left|\sum_{w \leqq s \leqq \frac{N}{t}} e\left(x\left(\frac{h_{1}}{(s t)^{j_{1}}}+\cdots+\frac{h_{r}}{(s t)^{j_{r}}}\right)\right)\right| \ll & \left(\frac{N}{t}\right)^{1-c \Lambda\left(\frac{N}{t}, x H\right)} \\
& +N^{j+1}(x t)^{-1}+H^{2}(\log x H)^{2 J}
\end{aligned}
$$

Therefore,

$$
\begin{aligned}
S_{1} \ll & \sum_{t \leqq U V}\left(\left(\frac{N}{t}\right)^{1-c \Lambda\left(\frac{N}{t}, x H\right)}+N^{j+1}(x t)^{-1}+H^{2}(\log x H)^{2 J}\right) \\
\ll & N^{1-c \Lambda\left(\frac{N}{U V}, x H\right)} \sum_{t \leqq U V} t^{-1+c \Lambda\left(\frac{N}{U V}, x H\right)}+N^{j+1} x^{-1} \sum_{t \leqq U V} \frac{1}{t}+U V H^{2}(\log x H)^{2 J} \\
\ll & N^{1-c \Lambda\left(\frac{N}{U V}, x H\right)} \Lambda\left(\frac{N}{U V}, x H\right)^{-1}(U V)^{c \Lambda\left(\frac{N}{U V}, x H\right)}+N^{j+1} x^{-1} \log U V \\
& +U V H^{2}(\log x H)^{2 J}
\end{aligned}
$$


For $U=V=N^{1 / 3}$ and $N \leqq x^{1 / j}$, this together with (28) implies according to Lemma 7

$$
\begin{aligned}
& \sum_{n \leqq N} \Lambda(n) e\left(x\left(\frac{h_{1}}{n^{j_{1}}}+\cdots+\frac{h_{r}}{n^{j_{r}}}\right)\right) \\
& \ll\left(N^{1-c \Lambda(N, x H)} \Lambda(N, x H)^{-1}+N^{j+1} x^{-1}+N^{\frac{2}{3}} H^{2}\right)(\log x H)^{2 J+1} \\
& \quad+N^{1 / 3}+\left(N^{1-c \Lambda(N, x H)}+N^{\frac{j+2}{2}} x^{-\frac{1}{2}}+N^{5 / 6} H^{\frac{1}{2}}\right)(\log x H)^{4 J} \\
& \ll\left(N^{1-c \Lambda(N, x H)}+N^{\frac{j+2}{2}} x^{-\frac{1}{2}}+N^{\frac{5}{6}} H^{2}\right)(\log x H)^{4 J} .
\end{aligned}
$$

Theorem 2. Let $2 \leqq N \leqq x^{1 / j}$. Then

$$
\sum_{p \leqq N} e\left(x\left(\frac{h_{1}}{p^{j_{1}}}+\cdots+\frac{h_{r}}{p^{j_{r}}}\right)\right) \ll\left(N^{1-c \Lambda(N, x H)}+N^{\frac{j+2}{2}} x^{-\frac{1}{2}}+N^{\frac{5}{6}} H^{2}\right)(\log x H)^{4 J} .
$$

Proof. By Chebyshev's theorem ([2], p. 55),

$$
\begin{aligned}
\sum_{n \leqq N} \Lambda(n) e\left(x\left(\frac{h_{1}}{n^{j_{1}}}+\cdots+\frac{h_{r}}{n^{j_{r}}}\right)\right) & =\sum_{\substack{p \\
p^{a} \leqq N}} \sum_{a} \log p e\left(x\left(\frac{h_{1}}{p^{a j_{1}}}+\cdots+\frac{h_{r}}{p^{a j_{r}}}\right)\right) \\
& =\sum_{p \leqq N} \log p e\left(x\left(\frac{h_{1}}{p^{j_{1}}}+\cdots+\frac{h_{r}}{p^{j_{r}}}\right)\right)+O(\log N \pi(\sqrt{N})) \\
& =\sum_{p \leqq N} \log p e\left(x\left(\frac{h_{1}}{p^{j_{1}}}+\cdots+\frac{h_{r}}{p^{j_{r}}}\right)\right)+O(\sqrt{N}) .
\end{aligned}
$$

Put

$$
h(N)=\left(N^{1-c \Lambda(N, x H)}+N^{\frac{i+2}{2}} x^{-\frac{1}{2}}+N^{\frac{5}{6}} H^{2}\right)(\log x H)^{4 J} .
$$

By partial summation, Lemma 8 and (30) give

$$
\begin{aligned}
\left|\sum_{p \leqq N} e\left(x\left(\frac{h_{1}}{p^{j_{1}}}+\cdots+\frac{h_{r}}{p^{j_{r}}}\right)\right)\right| \leqq & \left|\sum_{p \leqq N} \log p e\left(x\left(\frac{h_{1}}{p^{j_{1}}}+\cdots+\frac{h_{r}}{p^{j_{r}}}\right)\right)\right| \frac{1}{\log N} \\
& +\left|\int_{2}^{N} \sum_{p \leqq t} \log p e\left(x\left(\frac{h_{1}}{p^{j_{1}}}+\cdots+\frac{h_{r}}{p^{j_{r}}}\right)\right) \frac{d t}{t(\log t)^{2}}\right| \\
\ll & \frac{h(N)}{\log N}+\int_{2}^{N} \frac{h(t)}{t(\log t)^{2}} d t+\frac{\sqrt{N}}{\log N} \\
\ll & h(N)+(\log x H)^{4 J} \int_{2}^{N} t^{-c \Lambda(t, x H)} d t \\
& +(\log x H)^{4 J} x^{-1 / 2} \int_{2}^{N} t^{j / 2} d t+H^{2}(\log x H)^{4 J} \int_{2}^{N} t^{-\frac{1}{6}} d t
\end{aligned}
$$




$$
\begin{aligned}
& \ll h(N)+(\log x H)^{4 J}\left(\sqrt{N}+\int_{\sqrt{N}}^{N} t^{-c \Lambda(t, x H)} d t\right) \\
& \quad+\left(N^{\frac{j+2}{2}} x^{-\frac{1}{2}}+N^{\frac{5}{6}} H^{2}\right)(\log x H)^{4 J} \\
& \ll h(N)+(\log x H)^{4 J}\left(\sqrt{N}+N^{-\frac{1}{2} c A(\sqrt{N}, x H)} N\right) \\
& \ll h(N) .
\end{aligned}
$$

This completes the proof of Theorem 2 .

\section{Vinogradov's Fourier series method}

The following method may be found in [18], p. 32, or [1], Lemma 2.1 .

Let $0<\Delta<\frac{1}{4}$. For $J \in \mathbb{N}$ and real numbers $A_{j}$ and $B_{j}(1 \leqq j \leqq J)$ with

$$
0 \leqq B_{j}-A_{j} \leqq 1-2 \Delta
$$

there are 1-periodic functions $\psi_{j}(z)$, satisfying

$$
\psi_{j}(z)= \begin{cases}1 & \text { for } A_{j} \leqq z \leqq B_{j} \\ 0 & \text { for } B_{j}+\Delta \leqq z \leqq 1+A_{j}-\Delta\end{cases}
$$

and $0 \leqq \psi_{j}(z) \leqq 1$ for all $z$, such that

$$
\psi_{j}(z)=B_{j}-A_{j}+\Delta+\sum_{\substack{m=-\infty \\ m \neq 0}}^{\infty} a_{m, j} e(m z),
$$

where $a_{m, j} \in \mathbb{C}$ and for $|m|>0$ and $1 \leqq j \leqq J$

$$
\left|a_{m, j}\right| \ll \frac{1}{m^{2} \Delta}
$$

Theorem 3. Let $2 \leqq P \leqq x^{1 / J}, \underline{\sigma}=\left(\sigma_{1}, \ldots, \sigma_{J}\right)$ with $0<\sigma_{j} \leqq 1$ for $1 \leqq j \leqq J$ and

$$
D(\underline{\sigma})=D(\underline{\sigma} ; P, x)=\operatorname{card}\left\{p \leqq P:\left\{\frac{x}{p^{j}}\right\}<\sigma_{j}(1 \leqq j \leqq J)\right\}
$$

Then we have for arbitrary $\varepsilon>0$

$$
D(\underline{\sigma})=\sigma_{1} \cdots \sigma_{J} \pi(P)+O\left(P^{1-c \Lambda(P, x)}+P^{\frac{J+2}{2}+\varepsilon} x^{-\frac{1}{2}}\right)(\log x)^{4 J}
$$


Proof. For $\underline{A}=\left\{A_{1}, \ldots, A_{J}\right\}, \underline{B}=\left\{B_{1}, \ldots, B_{J}\right\}$, let

$$
T(\underline{A}, \underline{B})=\operatorname{card}\left\{p \leqq P: A_{j} \leqq\left\{\frac{x}{p^{j}}\right\} \leqq B_{j}(1 \leqq j \leqq J)\right\}
$$

Then

$$
T(\underline{A}, \underline{B}) \leqq \sum_{p \leqq P}\left(\sum_{j=1}^{J} \psi_{j}\left(\frac{x}{p^{j}}\right)\right) \leqq T(\underline{A}-\underline{A}, \underline{B}+\underline{A}),
$$

where $\underline{\Delta}=(\Delta, \ldots, \Delta)$. By $(31)$

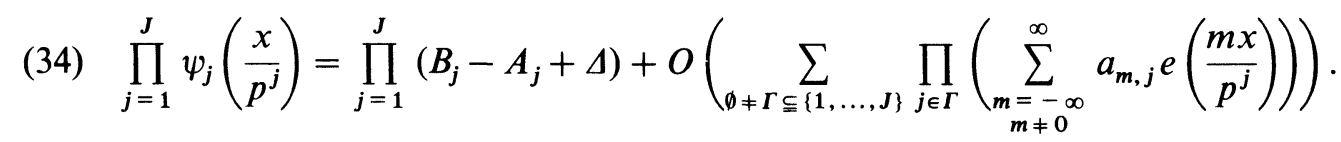

By (32),

$$
\text { (35) } \begin{aligned}
\sum_{\substack{m=-\infty \\
m \neq 0}}^{\infty} a_{m, j} e\left(\frac{m x}{p^{j}}\right) & =\sum_{0<|m|<\Delta^{-2}} a_{m, j} e\left(\frac{m x}{p^{j}}\right)+O\left(\sum_{|m| \geqq \Delta^{-2}} a_{m, j} e\left(\frac{m x}{p^{j}}\right)\right) \\
& =\sum_{0<|m|<\Delta^{-2}} a_{m, j} e\left(\frac{m n}{p^{j}}\right)+O\left(\sum_{|m| \geqq \Delta^{-2}} \frac{1}{m^{2} \Delta}\right) \\
& =\sum_{0<|m|<\Delta^{-2}} a_{m, j} e\left(\frac{m x}{p^{j}}\right)+O(\Delta) .
\end{aligned}
$$

Define $L$ to be the $O($ ) term in (34). The preceding equation yields

$$
\begin{aligned}
L & =\sum_{r=1}^{J} \sum_{1 \leqq j_{1}<\ldots<j_{r} \leqq J} \prod_{i=1}^{r}\left(\sum_{0<\left|m_{i}\right|<\Delta^{-2}} a_{m_{i}, j_{i}} e\left(\frac{m_{i} x}{p^{j_{i}}}\right)+O(\Delta)\right) \\
& \ll \sum_{r=1}^{J} \sum_{1 \leqq j_{1}<\ldots<j_{r} \leqq J} \sum_{i=1}^{r}\left(\sum_{0<\left|m_{i}\right|<\Delta^{-2}} a_{m_{i}, j_{i}} e\left(\frac{m_{i} x}{p^{j_{i}}}\right)\right)+O(\Delta) .
\end{aligned}
$$

Since $P \leqq x^{1 / J}$, we get by (32), (34) and Theorem 2

$$
\begin{aligned}
\sum_{p \leqq P}\left(\prod_{j=1}^{J} \psi_{j}\left(\frac{x}{p^{j}}\right)\right)= & \sum_{p \leqq P}\left(\prod_{j=1}^{J}\left(B_{j}-A_{j}+\Delta\right)\right) \\
& +O\left(\left.\max _{1 \leqq j_{1}<\ldots<j_{r} \leqq J}\right|_{0<\left|m_{1}\right|<\Delta^{-2}} \cdots \sum_{0<\left|m_{r}\right|<\Delta^{-2}} a_{m_{1}, j_{1}} \cdots a_{m_{r}, j_{r}}\right. \\
& \left.\quad \times \sum_{p \leqq P} e\left(x\left(\frac{m_{1}}{p^{j_{1}}}+\cdots+\frac{m_{r}}{p^{j_{r}}}\right)\right) \mid\right)+O(P \Delta) \\
= & \sum_{p \leqq P}\left(\prod_{j=1}^{J}\left(B_{j}-A_{j}+\Delta\right)\right) \\
& +O\left(\left(P^{1-c \Delta\left(P, x \Delta^{-2}\right)}+P^{\frac{J+2}{2}} x^{-\frac{1}{2}}+P^{\frac{5}{6}} \Delta^{-4}\right)\left(\log x \Delta^{-2}\right)^{4 J} \Delta^{-J}\right),
\end{aligned}
$$


if we choose for some suitable $\gamma=\gamma(J)>0$

$$
\Delta=P^{-\gamma \Lambda(P, x)} .
$$

At this point, we have to check that $\Delta<\frac{1}{4}$ as required by the initial conditions. This can be done by distinguishing two cases. If $(\log P)^{3} \ll(\log x)^{2}$, we have

$$
P^{1-c \Lambda(P, x)}(\log x)^{4 J} \gg P,
$$

hence the theorem obviously holds. Therefore, we may assume $(\log P)^{3} \gg(\log x)^{2}$, which yields the desired inequality for suitable $\gamma$. Then for arbitrarily small $\varepsilon>0$

$$
\begin{aligned}
\sum_{p \leqq P}\left(\prod_{j=1}^{J} \psi_{j}\left(\frac{x}{p^{i}}\right)\right)= & \left(\prod_{j=1}^{J}\left(B_{j}-A_{j}+\Delta\right)\right) \pi(P) \\
& +O\left(\left(P^{1-c \Lambda(P, x)}+P^{\frac{J+2}{2}+\varepsilon} x^{-\frac{1}{2}}+P^{\frac{5}{6}+\varepsilon}\right)(\log x)^{4 J}\right) \\
= & \left(\prod_{j=1}^{J}\left(B_{j}-A_{j}+\Delta\right)\right) \pi(P) \\
& +O\left(\left(P^{1-c \Lambda(P, x)}+P^{\frac{J+2}{2}+\varepsilon} x^{-\frac{1}{2}}\right)(\log x)^{4 J}\right) .
\end{aligned}
$$

Let $R$ denote the error term of the last equality. Then, by (33),

$$
T(\underline{A}, \underline{B}) \leqq \pi(P) \prod_{j=1}^{J}\left(B_{j}-A_{j}+\Delta\right)+|R|
$$

and

$$
T(\underline{A}-\underline{\Delta}, \underline{B}+\underline{\Delta}) \geqq \pi(P) \prod_{j=1}^{J}\left(B_{j}-A_{j}+\Delta\right)-|R| .
$$

Replacing $\underline{A}, \underline{B}$ by $\underline{A}-\underline{A}, \underline{A}$ resp. $\underline{B}, \underline{B}+\underline{A}$, (37) implies

$$
T(\underline{A}-\underline{\Delta}, \underline{A}) \leqq(2 \Delta)^{J} \pi(P)+|R|
$$

respectively

$$
T(\underline{B}, \underline{B}+\underline{\Delta}) \leqq(2 \Delta)^{J} \pi(P)+|R|
$$

Thus, by (38),

$$
\begin{aligned}
T(\underline{A}, \underline{B}) & =T(\underline{A}-\underline{\Delta}, \underline{B}+\underline{\Delta})-T(\underline{A}-\underline{\Delta}, \underline{A})-T(\underline{B}, \underline{B}+\underline{\Delta}) \\
& \geqq \pi(P) \prod_{j=1}^{J}\left(B_{j}-A_{j}+\Delta\right)-3|R|-2(2 \Delta)^{J} \pi(P) \\
& =\pi(P) \prod_{j=1}^{J}\left(B_{j}-A_{j}\right)+O\left(P^{1-c \Lambda(P, x)}\right)+O(R) \\
& =\pi(P) \prod_{j=1}^{J}\left(B_{j}-A_{j}\right)+O(R) .
\end{aligned}
$$


Similarly, we get by (37)

$$
T(\underline{A}, \underline{B}) \leqq \pi(P) \prod_{j=1}^{J}\left(B_{j}-A_{j}\right)+O(R) .
$$

Together, we have

$$
T(\underline{A}, \underline{B})=\pi(P) \prod_{j=1}^{J}\left(B_{j}-A_{j}\right)+O(R) .
$$

Setting $A_{j}=0, B_{j}=\sigma_{j}(1 \leqq j \leqq J)$, the desired result follows by observing that

$$
D(\underline{\sigma} ; P, x)=T(\underline{A}, \underline{B}) .
$$

\section{Proof of Theorem 1}

Let $m$ and $n$ be positive integers, and $p$ a prime. We define $U_{p}(m, n)$ to be the number of "carries" which occur when adding $m$ and $n$ in $p$-adic notation. Moreover, let $e(n ; p)=\max \left\{a \geqq 0: p^{a} \mid n\right\}$. An old result of Kummer is the following

Lemma 9 ([9], p. 116).

$$
e\left(\left(\frac{m+n}{m}\right) ; p\right)=U_{p}(m, n) .
$$

Now we are in the position to give the proof of Theorem 1. By (1),

$$
-\frac{m}{2}<-m^{1-\varepsilon}<m-2 k<m^{1-\varepsilon}<\frac{m}{2}
$$

for sufficiently large $m$. Hence

$$
m<\min (4 k, 4(m-k))
$$

Thus

$$
|m-2 k|<(\min (k, m-k))^{1-\varepsilon^{\prime}}
$$

for a suitable $\varepsilon^{\prime}>0$. Set

$$
n=\min (k, m-k), \quad d=|m-2 k| .
$$

Then

$$
\left(\begin{array}{c}
2 n+d \\
n
\end{array}\right)=\left(\begin{array}{l}
m \\
k
\end{array}\right)
$$


Let $\delta=\frac{\varepsilon^{\prime}}{3}$; let $J \in \mathbb{N}$ with $J>\frac{12 a}{\varepsilon^{\prime}}$. For sufficiently large $n$ and $K=\left[\frac{J}{1+\delta}\right]$, we get by Theorem 3 and the prime number theorem that

$$
\left\{n^{1 /(J+1)}<p<(2 n)^{1 /(J+1)}: \frac{2}{3}<\left\{\frac{n}{p^{j}}\right\}(K<j \leqq J),\left\{\frac{n}{p^{K}}\right\}<\frac{1}{2}\right\}
$$

is not empty, i.e. there is a prime $p$ satisfying

$$
\begin{gathered}
\frac{1}{2} p^{J+1}<n<p^{J+1} \\
\left\{\frac{n}{p^{j}}\right\}>\frac{2}{3} \quad(K<j \leqq J)
\end{gathered}
$$

and

$$
\left\{\frac{n}{p^{K}}\right\}<\frac{1}{2}
$$

Write $n$ in $p$-adic notation, namely

$$
n=n_{J} p^{J}+n_{J-1} p^{J-1}+\cdots+n_{1} p+n_{0} \quad\left(0 \leqq n_{j}<p\right) .
$$

For $K<j \leqq J$, we have by (43)

$$
\frac{2}{3}<\left\{\frac{n}{p^{j}}\right\}=\frac{n_{j-1} p^{j-1}+\cdots+n_{0}}{p^{j}}
$$

thus

$$
\frac{n_{j-1}}{p}>\frac{2}{3}-(p-1)\left(\frac{1}{p^{2}}+\cdots+\frac{1}{p^{j}}\right)>\frac{2}{3}-\frac{1}{p} .
$$

This implies for $p \geqq 7$

$$
n_{j-1}>\frac{1}{2} p
$$

i.e.

$$
n_{j}>\frac{1}{2} p \quad(K \leqq j<J) .
$$

By (44), we get in a similar fashion

$$
n_{K-1}<\frac{1}{2} p
$$

By (40) and (42), as well as the choice of $d, n$ and $J$,

$$
0 \leqq d=|m-2 k|<n^{1-\varepsilon^{\prime}}<p^{(J+1)\left(1-\varepsilon^{\prime}\right)}<p^{K-1} .
$$


Writing $d$ in $p$-adic notation, too, we therefore get

$$
d=d_{\mathbf{K}-2} p^{K-2}+\cdots+d_{0}
$$

with integers $d_{j}, 0 \leqq d_{j}<p$. Thus we have by (42), (45) and (46)

$$
\begin{aligned}
n & =n_{J} p^{J}+\cdots+n_{K-1} p^{K-1}+\cdots+n_{0}, \\
n+d & =n_{J}^{\prime} p^{J}+\cdots+n_{K-1}^{\prime} p^{K-1}+\cdots+n_{0}^{\prime},
\end{aligned}
$$

where

$$
n_{j}=n_{j}^{\prime}>\frac{1}{2} p \quad(K \leqq j \leqq J)
$$

By Lemma 9,

$$
e\left(\left(\begin{array}{c}
2 n+d \\
n
\end{array}\right) ; p\right) \geqq J-K \geqq a,
$$
which means that there is a $p$ satisfying $p^{a} \mid\left(\begin{array}{c}2 n+d \\ n\end{array}\right)$. By the definition of $n$ and (39)
we have

$$
n>\frac{1}{4} m
$$

thus by (42)

$$
p>\left(\frac{m}{4}\right)^{\frac{1}{J+1}} \geqq \frac{1}{2} m^{\frac{1}{J+1}}
$$

By (41), the proof of Theorem 1 is completed.

\section{References}

[1] R.C. Baker, Diophantine Inequalities, Oxford 1986.

[2] H. Davenport, Multiplicative Number Theory, 2nd edition, New York-Heidelberg-Berlin 1980.

[3] P. Erdös, R.L. Graham, On the prime factors of $\left(\begin{array}{l}n \\ k\end{array}\right)$, Fibonacci Quarterly 14 (1976), 348-352.

[4] P. Erdös, R.L. Graham, Old and New Problems and Results in Combinatorial Number Theory, L'Enseign. Math., Geneva 1980.

[5] P. Erdös, Problems and Results on Number Theoretic Properties of Consecutive Integers and Related Questions, Proc. Fifth Manitoba Conf. on Numerical Mathematics (1975), 25-44.

[6] R.K. Guy, Unsolved Problems in Number Theory, New York-Heidelberg-Berlin 1981.

[7] M. Jutila, On the numbers with a large prime factor, II, J. Indian Math. Soc. 38 (1974), 125-130.

[8] A.A. Karacuba, Estimates for trigonometric sums by Vinogradov's method, and some applications, Proc. Steklov Inst. Math. 112 (1971), 251-265.

[9] E. E. Kummer, Über die Ergänzungssätze zu den allgemeinen Reciprocitätsgesetzen, J. reine angew. Math. 44 (1852), 93-146.

[10] J.W. Sander, On prime powers dividing $\left(\begin{array}{c}2 n \\ n\end{array}\right)$, to appear.

[11] J.W. Sander, Prime power divisors of $\left(\begin{array}{c}2 n \\ n\end{array}\right)$, J. Numb. Th. 39 (1991), 65-74. 
[12] J.W. Sander, On numbers with a large prime power factor, Acta Math. Hung., to appear.

[13] J.W. Sander, On prime divisors of binomial coefficients, Bull. London Math. Soc., to appear.

[14] A. Sárközy, On Divisors of Binomial Coefficients, I, J. Numb. Th. 20 (1985), 70-80.

[15] E.C. Titchmarsh, The Theory of the Riemann Zeta-function, 2nd edition, Oxford 1986.

[16] R.C. Vaughan, Sommes trigonométriques sur les nombres premiers, Comptes Rendus Acad. Sci. Paris 285 (1977), 981-983.

[17] R.C. Vaughan, On the distribution of $\alpha p$ modulo 1, Mathematika 24 (1977), 135-141.

[18] I.M. Vinogradov, The Method of Trigonometrical Sums in the Theory of Numbers, London.

Institut für Mathematik, Universität Hannover, Welfengarten 1, D-W-3000 Hannover 1

Eingegangen 4. Januar 1991 